\title{
Pelvic pain and early IUD discontinuation: a prospective cohort study
}

\author{
Sharon R. Gerber ${ }^{1 *}$, Noah Nattell ${ }^{1}$, Nora Doty ${ }^{1}$, \\ Xiaoyu Liu ${ }^{2}$, Jessica R. Overbey ${ }^{2}$, Rachel Masch ${ }^{1}$
}

${ }^{1}$ Department of Obstetrics, Gynecology and Reproductive Science, ${ }^{2}$ Department of Population Health Science and Policy, Icahn School of Medicine at Mount Sinai, New York, USA

Received: 12 November 2018

Accepted: 05 February 2019

\section{*Correspondence:}

Dr. Sharon R. Gerber,

E-mail: sharon.gerber@mssm.edu

Copyright: (c) the author(s), publisher and licensee Medip Academy. This is an open-access article distributed under the terms of the Creative Commons Attribution Non-Commercial License, which permits unrestricted non-commercial use, distribution, and reproduction in any medium, provided the original work is properly cited.

\section{ABSTRACT}

Background: The aim of the study is to assess if pelvic pain is a risk factor for intrauterine device (IUD) discontinuation within one year of placement.

Methods: This is a prospective cohort study of women who had IUDs inserted at a family planning office for the primary intent of contraception. Baseline pelvic pain characteristics were assessed using a validated pelvic pain questionnaire. Women were contacted at 1 year to assess IUD continuation.

Results: From February 1, 2014 to August 11, 2015 authors enrolled a sample of 179 women. Of the 179 enrolled,163 participants completed the questionnaire, 98 reported a history of baseline pelvic pain and 65 reported no history of baseline pelvic pain. 20 participants were lost to follow-up. 86 women in the pelvic pain and 57 in the no pelvic pain group were included in the final analysis. Discontinuation rates at one year follow up were $25.6 \%(22)$ and $35.1 \%$ (20) respectively. There was no significant difference in those with and without pelvic pain discontinuing IUDs at one year $(\mathrm{p}=0.22)$.

Conclusions: Baseline generalized pelvic pain may not be a risk factor for IUD discontinuation within one year of placement.

Keywords: Intrauterine device (IUD), Pelvic pain, Risk factor

\section{INTRODUCTION}

Intrauterine devices (IUDs) are a form of long-acting reversible contraception or LARC. They are an effective and important public health tool to reduce unintended pregnancy and subsequently improve the welfare of women and girls. The Contraceptive CHOICE Project demonstrated the enormous impact of LARC when access is improved by removing cost-barriers and providing standardized patient education. ${ }^{1}$

The CHOICE project also revealed that LARC users of all age groups are more likely to continue using their chosen method at one year. Despite a continuation rate of $85 \%$ for both the copper (CuT380A) and 52-mg
levonorgestrel-IUDs (LNG-IUD), there are certain common complaints associated with early discontinuation. $^{2}$

Those that underwent early IUD removal in the CHOICE project cited cramping to be the number one reason for discontinuation. ${ }^{3}$ A secondary analysis of a clinical trial of lidocaine gel use during IUD insertion utilized chart review to determine if baseline dysmenorrhea predicted IUD discontinuation at 1 year. Researchers found that women who discontinued the IUD had higher baseline dysmenorrhea scores than those who continued. ${ }^{4}$

There is a high known prevalence of baseline pelvic pain. In a multi-country, systematic review conducted by the 
World Health Organization (WHO), estimates of pelvic pain varied widely: between 16 to $81 \%$ for dysmenorrhea, 8 to $21.8 \%$ for dyspareunia and 2.1 to $24 \%$ for non-cyclic pelvic pain. ${ }^{5,6}$

Given the large upfront expense of IUDs, it would be cost-effective to better understand if baseline characteristics can predict early discontinuation. ${ }^{7}$

Predicting risk factors for early discontinuation can also help healthcare providers to better counsel their patients. The objective of this study is to assess if generalized pelvic pain is a risk factor for IUD discontinuation within one year of placement.

\section{METHODS}

This is a prospective, observational study that occurred at a hospital-based family planning office in New York City with institutional review board approval. Patients were enrolled between February 1, 2014 and August 11, 2015.

\section{Inclusion criteria}

- English-speaking women, aged 18 years and older, who presented to the family planning office for IUD insertion for the primary intent of contraception were eligible. Women chose which type of IUD they wanted.

\section{Exclusion criteria}

- Women who had a current IUD in place or their primary reason for IUD placement was for a reason other than contraception.

The objective of this study was to determine if baseline pelvic pain and dysmenorrhea influenced IUD discontinuation rates.

The study was initially powered with the assumption of a baseline prevalence of dysmenorrhea and pelvic pain of $15 \%$. 5 Based on previously published data, authors expected a $10-15 \%$ discontinuation rate within 1 year. ${ }^{2}$

In order to determine a $10 \%$ difference in discontinuation between groups with and without pelvic pain, with a power of $80 \%$ and alpha of 0.05 , authors estimated the need to enrol 215 subjects.

Authors anticipated a $25 \%$ loss to follow-up and thus our goal was to recruit 300 participants.

Enrollment was slower than anticipated because of loss of study personnel.

Therefore, after 18 months, study enrollment was stopped and data was analysed. Originally, authors had intended to look at dysmenorrhea separately from other types of pelvic pain (non-cyclic pain and dyspareunia), but these pelvic pain baseline characteristics overlapped in our population.

Due to lower than expected recruitment and overlap in quality of pelvic pain, the decision was made to combine the groups and analyse all subjects who reported any type of pelvic pain into one cohort.

The rates of IUD discontinuation at 1 year in subjects with and without pelvic pain were compared using a Chisquare test.

Baseline characteristics and the reason for IUD removal were compared between the groups using T-tests, Chisquare or Fisher's exact tests as appropriate. All analyses were conducted using SAS 9.4 (SAS Institute, Cary, NC).

\section{RESULTS}

From February 1, 2014 to August 11, 2015 authors enrolled 179 participants. Of the 179 participants enrolled, 163 completed the questionnaire and were included in our analysis. Of the 163 participants, 98 $(60.1 \%)$ reported a history of pelvic pain and $65(39.9 \%)$ reported no history of pelvic pain.

Most subjects were white, cohabitating with a partner, and high school graduates. Participants with a history of pelvic pain were more likely to be single and nulliparous (Table 1). Participants were more likely to choose a hormonal IUD regardless of pelvic pain history (Figure 1).

Of the 163 patients that completed the baseline survey, 20 were lost to follow up at one year. Participants lost to follow up were younger, more likely to have given birth in the previous year and less likely to be working full or part time.

Of the 143 women reached for follow-up at 1 year, 86 had reported pelvic pain at baseline.

Twenty-two subjects $(25.6 \%)$ in the group with baseline pelvic pain and $20(35.1 \%)$ in the group without pelvic pain discontinued IUDs at 1 year.

Participants with a history of pelvic pain were 0.73 times less likely to have their IUD removed compared to those with no history of pelvic pain (95\% CI: $0.44,1.21)$. This was not statistically significant $(\mathrm{p}=.22)$. (Table 2$)$.

Reason for IUD discontinuation was ascertained for 35 of the 42 women that reported IUD discontinuation. Most of these women attributed removal to either bleeding $(n=10)$ or pain $(n=8)$ followed by desire for pregnancy $(n=6)$. Other reasons included malpositioned IUD $(n=5)$, IUD expulsion $(n=5)$ and one report of pregnancy with IUD in situ. 
Table 1: Patient characteristics.

\begin{tabular}{|c|c|c|c|c|c|c|c|}
\hline & \multicolumn{2}{|c|}{ All $(n=163)$} & \multicolumn{2}{|c|}{ Pelvic pain $(\mathrm{n}=98)$} & \multicolumn{2}{|c|}{$\begin{array}{l}\text { No pelvic pain } \\
(\mathrm{n}=65)\end{array}$} & \multirow[t]{2}{*}{ P-value } \\
\hline & No. & Mean \pm SD & No. & Mean \pm SD & No. & Mean \pm SD & \\
\hline Age (years) & 160 & $31.1 \pm 6.9$ & 95 & $31.4 \pm 7.4$ & 65 & $30.6 \pm 6.2$ & 0.45 \\
\hline Height (inches) & 161 & $64.3 \pm 2.9$ & 96 & $64.4 \pm 2.8$ & 65 & $64.1 \pm 3.0$ & 0.64 \\
\hline Weight (pounds) & 159 & $151.2 \pm 37.8$ & 96 & $153.2 \pm 35.9$ & 63 & $148.3 \pm 40.6$ & 0.42 \\
\hline BMI & 159 & $25.8 \pm 6.2$ & 96 & $26.0 \pm 5.8$ & 63 & $25.4 \pm 6.8$ & 0.52 \\
\hline Number of children & 157 & $1.2 \pm 1.3$ & 94 & $1.0 \pm 1.4$ & 63 & $1.3 \pm 1.2$ & 0.16 \\
\hline Number of children born by cesarean section & 158 & $0.3 \pm 0.8$ & 95 & $0.3 \pm 0.9$ & 63 & $0.2 \pm 0.6$ & 0.69 \\
\hline Marital status & \multicolumn{2}{|c|}{ No. $(\%)$} & \multicolumn{2}{|c|}{ No. $(\%)$} & \multicolumn{2}{|c|}{ No. $(\%)$} & \\
\hline Single and living with a partner & \multicolumn{2}{|c|}{$47(29.2)$} & \multicolumn{2}{|c|}{$28(29.2)$} & \multicolumn{2}{|c|}{$19(29.2)$} & 0.0007 \\
\hline Married & \multicolumn{2}{|c|}{$51(31.7)$} & \multicolumn{2}{|c|}{$20(20.8)$} & \multicolumn{2}{|c|}{$31(47.7)$} & \\
\hline Single (not living with a partner) & \multicolumn{2}{|c|}{$57(35.4)$} & \multicolumn{2}{|c|}{$44(45.8)$} & \multicolumn{2}{|c|}{$13(20.0)$} & \\
\hline Divorced or separated & \multicolumn{2}{|c|}{$6(3.7)$} & \multicolumn{2}{|c|}{$4(4.2)$} & \multicolumn{2}{|c|}{$2(3.1)$} & \\
\hline Widowed & \multicolumn{2}{|c|}{0} & \multicolumn{2}{|c|}{0} & \multicolumn{2}{|c|}{0} & \\
\hline \multicolumn{8}{|l|}{ Education } \\
\hline Some high school or less & \multicolumn{2}{|c|}{$6(3.8)$} & \multicolumn{2}{|c|}{$2(2.1)$} & $4(6$. & & 0.15 \\
\hline High school graduate & 23( & & $12(1$ & $.5)$ & $11(1$ & 7) & \\
\hline Some college & 21( & & $12(1$ & $.5)$ & $9(1<$ & & \\
\hline Associates degree (2-year degree) & $13(\varepsilon$ & & $5(5$. & & $8(12$ & & \\
\hline Bachelor's degree (4-year degree) & $62(3$ & & 39( & .6) & $23(3$ & $59)$ & \\
\hline Masters or professional degree & $35(2$ & & $26(2$ & $.1)$ & $9(1<$ & & \\
\hline Ethnicity & & & & & & & \\
\hline $\begin{array}{l}\text { American Indian, Native American or } \\
\text { Alaska native }\end{array}$ & $2(1$. & & $2(2$. & & 0 & & 0.031 \\
\hline Asian (Far East or Southeast Asian) & $9(5$. & & $3(3$. & & $6(9$. & & \\
\hline Asian (South Asian or Indian subcontinent) & $1(0$. & & 0 & & $1(1$. & & \\
\hline Black or African America (not Hispanic) & 18 & & $12(1$ & & $6(9$. & & \\
\hline Hispanic or Latina & $52(3$ & & $24(2$ & $3)$ & $28(4$ & $3.1)$ & \\
\hline White (not Hispanic) & 65( & & 46( & & $19(2$ & .2) & \\
\hline Mixed Race & $13(\varepsilon$ & & $8(8$. & & $5(7$. & & \\
\hline Ever pregnant & & & & & & & 0.0012 \\
\hline Yes & 110 & & $56(6$ & & $54(\varepsilon$ & 1.4) & \\
\hline No & $47(2$ & & $37(3$ & & $10(1$ & .6) & \\
\hline Pregnant in the last 12 months & & & & & & & \\
\hline Yes & $64(4$ & & $31(3$ & & $33(5$ & $3.2)$ & 0.014 \\
\hline No & $91(5$ & & $62(6$ & & $29(4$ & $6.8)$ & \\
\hline Dilation and curettage & & & & & & & \\
\hline Yes & 50 & & $31(3$ & & $19(2$ & $.7)$ & 0.73 \\
\hline No & 110 & & $65(6$ & & $45(7$ & $.3)$ & \\
\hline Ectopic pregnancy & & & & & & & \\
\hline Yes & $7(4$. & & $4(4$. & & $3(4$. & & $>0.99$ \\
\hline No & 153 & & $92(c$ & .8) & $61(c$ & .3) & \\
\hline
\end{tabular}

Table 2: IUD removal and reasons.

\begin{tabular}{|c|c|c|c|c|}
\hline & All $(n=143)$ & Pelvic Pain (n=86) & No Pelvic Pain $(\mathrm{n}=57)$ & P value \\
\hline & No. $(\%)$ & No. $(\%)$ & No. $(\%)$ & \\
\hline IUD removed & $42(29.4)$ & $22(25.6)$ & $20(35.1)$ & 0.22 \\
\hline & All $(n=35)$ & Pelvic pain $(n=20)$ & No pelvic pain $(n=15)$ & $P$ value \\
\hline IUD removed reason & No. $(\%)$ & No. $(\%)$ & No. $(\%)$ & \\
\hline Became pregnant & $1(2.9)$ & $1(5.0)$ & 0 & 0.99 \\
\hline Wanted to become pregnant & $6(17.1)$ & $3(15.0)$ & $3(20.0)$ & \\
\hline Bleeding & $10(28.6)$ & $5(25.0)$ & $5(33.3)$ & \\
\hline Pain & $8(22.9)$ & $5(25.0)$ & $3(20)$ & \\
\hline Malpositioned IUD & $5(14.3)$ & $3(15.0)$ & $2(13.3)$ & \\
\hline Expulsion & $5(14.3)$ & $3(15.0)$ & $2(13.3)$ & \\
\hline
\end{tabular}




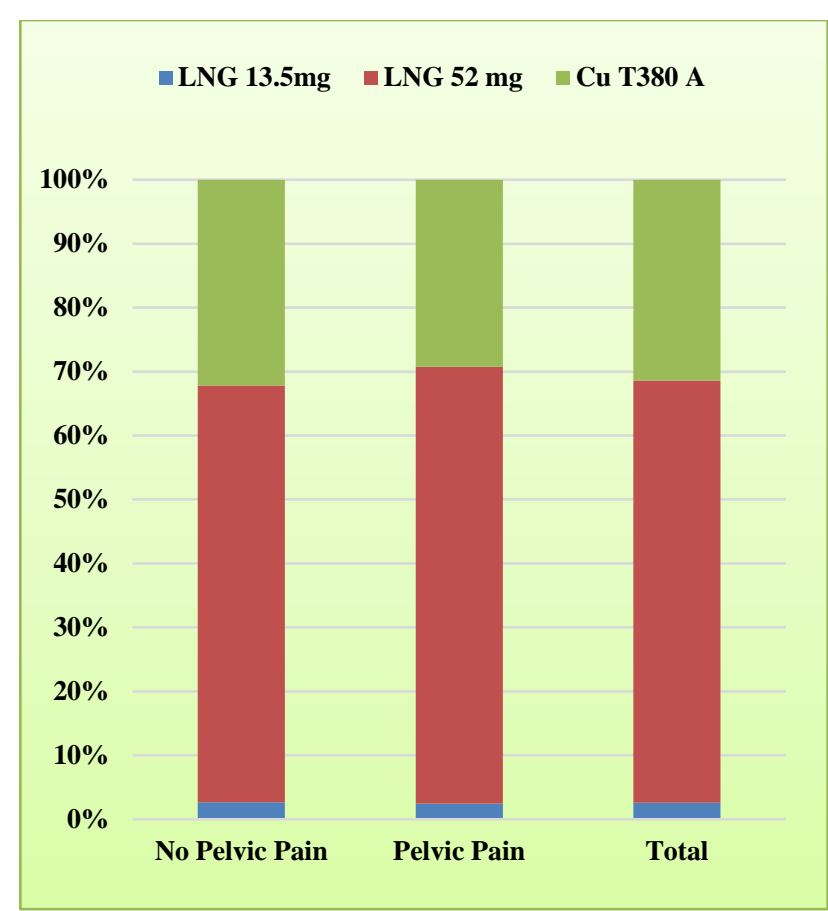

Figure 1: IUD Selection by presence of baseline pelvic pain.

\section{DISCUSSION}

The goal of this study was to ascertain if a history of pelvic pain could predict early discontinuation of IUDs. Authors wanted to recruit 300 participants during the study period in order to detect a $10 \%$ difference in early IUD discontinuation between women with and without a history of pelvic pain. This recruitment goal was based on the assumption of a $25 \%$ loss to follow up and a $15 \%$ prevalence of pelvic pain. The prevalence of pelvic pain in our cohort was 29\%. This larger prevalence theoretically increases the power to detect a difference, yet no statistically significant difference was detected. In this study, there was a trend for participants with a history of pelvic pain to be less likely to discontinue their IUDs at the one-year mark. While, our results demonstrate no statistically significant difference in this primary outcome, further research with a larger cohort is necessary to validate these findings.

Authors hypothesize that the higher prevalence of pelvic pain in present study was due to our decision to combine dysmenorrhea, dyspareunia and noncyclic pelvic pain into one pelvic pain category secondary to slow recruitment. Based on the WHO systematic review, there is a moderately wide range for dyspareunia and noncyclic pelvic pain, 8 to $21.8 \%$ and 2.1 to $24 \%$, respectively, leading to a $15 \%$ prevalence assumption. The inclusion of dysmenorrhea with a vastly wide prevalence range from $16 \%$ to $81 \%$, likely raised the prevalence among present study participants. ${ }^{6}$

Previous research has indicated that higher dysmenorrhea scores may be associated with early IUD removal. In the aforementioned Maguire study, which analyzed a prospective cohort of 199 IUD recipients, women with early IUD discontinuation had higher median dysmenorrhea scores before insertion than those who continued (42 vs. 25, p=.03) [4]. Women in Maguire's study predominately chose the $\mathrm{Cu}$ T380A which is known to be associated with increased bleeding and cramping during menses. While $71 \%$ of women in the early discontinuation group were $\mathrm{Cu}$ T380A users, given the popularity of $\mathrm{Cu}$ T380A in this cohort, this was not statistically significant. Unlike the Maguire study, participants in present study predominantly chose hormonal IUDs which is known to be a treatment for cyclic pelvic pain associated with dysmenorrhea, endometriosis and adenomyosis..$^{8,9,10}$ The popularity of hormonal IUDs in present study may have contributed to the higher rate of IUD retention among participants with a history of pelvic pain, although not statistically significant. Additionally, unlike Maguire et al., present study did not differentiate dysmenorrhea from chronic and non-cyclic forms of pelvic pain.

Other studies have explored the reasons for discontinuation of both copper and hormonal IUDs, siting pelvic pain as a primary reason for discontinuation. ${ }^{11,12} \mathrm{In}$ a prospective study on IUD continuation among nulliparous women, the majority of participants, like participants in this study, chose the hormonal IUD. Baseline dysmenorrhea was similar in both hormonal and copper IUD groups. 1-year follow up data of 109 women (88 LNG-IUS and $21 \mathrm{Cu}$ T 380A users) reported 5 users who discontinued the IUD for pelvic pain, 3 hormonal IUD users and 2 copper IUD users respectively. An additional 8 users continued with the IUD despite pelvic pain related to pain with intercourse ( 2 users) and menstrual cramping ( 5 users). ${ }^{11}$ Of the participants in present study contacted for follow up, reasons for early discontinuation were obtained for 35 participants. 8 participants $(22.9 \%)$ attributed discontinuation to pain. Five of these participants were in the pelvic pain group and three in the group without pelvic pain. The popularity of hormonal IUDs in both of these studies would likely play a role in the low number of participants in the early discontinuation group citing pain as a reason for discontinuation. Alternatively, a study on continuation rates in a cohort using only the copper IUD, reported that pelvic pain was experienced by 34 or 107 women contacted for follow up. Interestingly, however, only 8 participants discontinued the IUD for this reason. ${ }^{12}$ Overall IUD continuation rates at 1-year are high despite side effects, $70.6 \%$ in present study, $83 \%$ and $79.1 \%$ in the study with both copper and hormonal IUD cohorts and the copper only cohort study respectively.

Strengths of this study include that it is a prospective study with phone contact within 13 months of IUD insertion to ascertain whether the IUD was discontinued early and if so, the reason for discontinuation. Another strength is that a validated pelvic pain questionnaire was used to determine baseline pelvic pain in participants and 
thus captured women who experience different types of pelvic pain. Limitations of this study include recall bias for those who had IUD removed months prior to contact, slow enrollment, loss to follow-up and changes in study personnel over the course of the study. Also, secondary to overlapping pelvic pain characteristic in our population and slow enrollment, authors did not have enough participants to adequately differentiate dysmenorrhea from noncyclic forms of pelvic pain. In this prospective cohort study, a history of pelvic pain was not a predictor of early IUD discontinuation. Future studies to confirm these results may be used as guidance to clinicians and their patients. Further areas of investigation include determining whether IUD type (hormonal vs. copper) influences early discontinuation in patients with a history of pelvic pain as well as discerning type of pelvic pain (i.e. dysmenorrhea, chronic pelvic pain, dyspareunia) and its influence on early IUD discontinuation.

\section{CONCLUSION}

IUDs are acceptable among women with and without a history of pelvic pain. A history of pelvic pain may not be associated with early IUD discontinuation. More research is needed to determine which factors are associated with early IUD discontinuation.

Funding: No funding sources Conflict of interest: None declared

Ethical approval: The study was approved by the Institutional Ethics Committee

\section{REFERENCES}

1. McNicholas C, Tessa M, Secura G, Peipert JF. The contraceptive CHOICE project round up: what we did and what we learned. Clinic Obstet Gynecol. 2014;57(4):635.

2. Rosenstock JR, Peipert JF, Madden T, Zhao Q, Secura GM. Continuation of reversible contraception in teenagers and young women. Obstet Gynecol. 2012;120(6): 1298.

3. Grunloh DS, Casner T, Secura GM, Peipert JF, Madden T. Characteristics associated with discontinuation of long-acting reversible contraception within the first 6 months of use. Obstet Gynecol. 2013;122(6):1214-21.

4. Maguire K, Joslin-Roher S, Westhoff CL, Davis AR. IUDs at 1 year: predictors of early discontinuation. Contracep. 2015;92(6):575-7.

5. Zondervan KT, Yudkin PL, Vessey MP, Jenkinson CP, Dawes MG, Barlow DH, et al. The community prevalence of chronic pelvic pain in women and associated illness behaviour. $\mathrm{Br} \mathrm{J}$ Gen Pract. 2001;51(468):541-7.

6. Latthe P, Latthe M, Say L, Gülmezoglu M, Khan KS. WHO systematic review of prevalence of chronic pelvic pain: a neglected reproductive health morbidity. BMC Public Health. 2006;6(1):177.

7. Eisenberg D, McNicholas C, Peipert JF. Cost as a barrier to long-acting reversible contraceptive (LARC) use in adolescents. J Adolescent Health. 2013;52(4):S59-63.

8. Imai A, Matsunami $\mathrm{K}$, Takagi $\mathrm{H}$, Ichigo $\mathrm{S}$. Levonorgestrel-releasing intrauterine device used for dysmenorrhea: five-year literature review. Clinic Experiment Obstet Gynecol. 2014;41(5):495-8.

9. Rafique S, Decherney AH. Medical Management of Endometriosis. Clinic Obstet Gynecol. 2017;60(3):485-96.

10. Radzinsky VE, Khamoshina MB, Nosenko EN, Dukhin AO, Sojunov MA, Orazmuradov AA, Lebedeva MG, Orazov MR. Treatment strategies for pelvic pain associated with adenomyosis. Gynecol Endocrinol. 2016;32(2):19-22.

11. Hall AM, Kutler BA. Intrauterine contraception in nulliparous women: a prospective survey. J Fam Plann Reprod Health Care. 2016 ;42(1):36-42.

12. Bateson D, Harvey C, Trinh L, Stewart M, Black KI. User characteristics, experiences and continuation rates of copper intrauterine device use in a cohort of Australian women. Aus New Zealand J Obstet Gynaecol. 2016;56(6):655-61.

Cite this article as: Gerber SR, Nattell N, Doty N, Liu X, Overbey JR, Masch R. Pelvic pain and early IUD discontinuation: a prospective cohort study. Int J Reprod Contracept Obstet Gynecol 2019;8:792-6. 